\title{
Early Clinical Expressions of Insulin Resistance: The Real Enemy to Look For
}

\author{
René Rodríguez-Gutiérrez • Alejandro Salcido-Montenegro · José Gerardo González-González
}

Received: October 16, 2017 / Published online: December 5, 2017

(C) The Author(s) 2017. This article is an open access publication

\section{ABSTRACT}

The type 2 diabetes mellitus epidemic threatens public healthcare systems worldwide. Efforts to prevent chronic complications of diabetes and reduce their associated mortality have been ineffective. Hence, early prevention of type 2 diabetes mellitus and cardiovascular disease needs to be prioritized. This strategy, however, must be centered not on an approach based on hyperglycemia but on early pathophysiologic

Enhanced content To view enhanced content for this article, go to http://www.medengine.com/Redeem/ 15FCF0602131E08C.

R. Rodríguez-Gutiérrez · A. Salcido-Montenegro · J. G. González-González ( $\square$ )

Endocrinology Division, Department of Internal

Medicine, University Hospital, Universidad

Autonoma de Nuevo Leon, Monterrey 64460,

Mexico

e-mail: jgerardo@meduanl.com

R. Rodríguez-Gutiérrez

Knowledge and Evaluation Research Unit in

Endocrinology, Mayo Clinic, Rochester, MN 55905, USA

R. Rodríguez-Gutiérrez

Division of Endocrinology, Diabetes, Metabolism and Nutrition, Department of Medicine, Mayo Clinic, Rochester, MN 55905, USA

J. G. González-González

Research Unit, University Hospital “Dr. José E.

González", Universidad Autonoma de Nuevo Leon,

Monterrey 64460, Mexico mechanisms, such as insulin resistance. Nonalcoholic fatty liver disease, androgenic alopecia, acanthosis nigricans, and polycystic ovarian syndrome are all well-accepted early clinical manifestations of insulin resistance that represent, in themselves, a risk for further development of type 2 diabetes and that appear years before hyperglycemia. Therefore, focusing efforts on detecting and rigorously treating patients with early clinical expression of insulin resistance (insulin resistance clinical syndrome) is probably the course of action that needs to be taken to counterbalance the type 2 diabetes mellitus epidemic.

Keywords: Insulin resistance; Type 2 diabetes mellitus; Polycystic ovarian syndrome; Acanthosis nigricans; Non-alcoholic fatty liver disease; Androgenic alopecia

\section{CLINICAL VIGNETTE}

Today, a very common clinical scenario is a 17-year-old female with a family history of type 2 diabetes mellitus (T2DM) and hypertension in her mother and two first-degree relatives. Three years ago she was diagnosed with polycystic ovarian syndrome (PCOS). She is overweight with her body weight increasing $10 \mathrm{~kg}$ in the last 2 years. Additionally, she has acanthosis nigricans (AN) in the axillae and knuckles. 
Blood tests were unremarkable with a fasting plasma glucose value of $82 \mathrm{mg} / \mathrm{dl}$ and an HbA1c of $5.0 \%$.

The first question of this clinical vignette is: does this young woman have T2DM? The answer is, of course, no. The second question is: does she have insulin resistance (IR) with compensatory hyperinsulinemia? The answer is yes. Currently, however, the approach endorses a delay of one to two decades to establish a diagnosis of T2DM. Based on this everyday clinical case and the predictable medical actions recommended for the patient, the next question is: do we really understand which is the initial true time to act against T2DM? Should it be with clinical expression of IR or hyperglycemia?

\section{INTRODUCTION}

Previous projections of T2DM prevalence have been exceeded, with a current worldwide prevalence of more than 435 million patients [1]. The socioeconomic implications of this epidemic threaten worldwide public health systems, and epidemiologic statistics of T2DM/obesity are familiar to all clinicians. In fact, there has been a 100 million increment in T2DM cases in the last 10 years and an annual death rate of 1.5 million [2]. Three out of four patients with T2DM are economically active and more than $30 \%$ of the global economic burden estimated for T2DM is due to indirect expenses with mortality as the main associated cause [3]. The current projections are of catastrophic socioeconomic consequences and require an immediate call for action. The T2DM epidemic requires a different medical approach. Efforts to prevent major macrovascular complications and reduce their mortality have been ineffective [4]. Antidiabetic medications that have shown evidence of macrovascular risk reduction have been studied only in very high-risk cardiovascular patients with T2DM, and, unfortunately, these drugs are not easily available for many patients, particularly in countries with a very high incidence of T2DM, such as in developing countries [5]. As a consequence, the main emphasis must focus on very early prevention of T2DM and cardiovascular disease (CVD). This strategy, however, must be centered not on the development of hyperglycemia but on the disorders occurring as a result of earlier pathophysiological mechanisms, such as IR. Its manifestations are tissue-specific signs that arise as the metabolic burden worsens, some even before the development of weight gain or obesity [6-10]. Despite the absence of consensus in the diagnosis and management of IR, there is no question that T2DM, hypertension, obesity, non-alcoholic fatty liver disease (NAFLD), androgenetic alopecia, and AN are well-recognized clinical expressions of IR, particularly when they occur as a cluster and are associated with a family history of the same.

This article is based on previously conducted studies and does not involve any new studies of human or animal subjects performed by any of the authors.

\section{EARLY CLINICAL EXPRESSIONS OF INSULIN RESISTANCE}

AN, classically described in the neck, axillae, and elbows of patients with obesity, has been recently reported as an early and straightforward marker of IR in young patients even with a normal BMI, mainly in an ignored site such as the knuckles. It has potential value as a useful tool for screening patients with a high-metabolic risk in early stages of the disease [6]. Patients with PCOS have a higher risk for developing gestational diabetes $(\mathrm{OR}=2.1)$ and $\mathrm{T} 2 \mathrm{DM}(\mathrm{OR}=8.8)$ and a higher prevalence of T2DM. Furthermore, when added to AN, the prevalence of T2DM increases [7, 8]. Early androgenetic alopecia has been suggested as a clinical marker of IR, as well as presenting an increased CVD risk [9]. NAFLD has been associated with IR and is an early detectable marker of metabolic disease. It is found most frequently in women with gestational diabetes mellitus. Patients with NAFLD present a three- to fourfold increased risk of developing T2DM [10].

\section{ACT EARLIER-INSULIN RESISTANCE CLINICAL SYNDROME}

We recommend a T2DM approach not centered on hyperglycemia but on strict management of well-accepted IR clinical expressions. 
Furthermore, we could go a step further and develop an easy and clear name to identify clinical expressions of IR, such as IR clinical syndrome. Therapy should be as strict as proposed today once postprandial or fasting hyperglycemia occurs. Under this perspective, the main disorder to start therapy must be IR clinical expressions (e.g., AN, androgenic alopecia, PCOS). As soon as they appear, instead of delaying therapy until the diagnosis of T2DM, obesity, hypertension, dyslipidemia, or CVD, all these individuals should undergo an intensive aerobic and anaerobic exercise program, a weight control low-calorie diet, and likely for some metformin therapy (i.e., highrisk patients with other comorbidities) under close surveillance.

Treating only an elevated IR laboratory index could be imprecise and lead to the other side of the spectrum, treating surrogate markers rather than patients, a common practice nowadays that diminishes resources and diverts attention from those who could really benefit the most. However, when several IR clinical manifestations are taken into account, it is difficult to ignore that these are patients who will develop obesity, metabolic syndrome, T2DM, and CVD in the medium term. Our lack of a strong action in this scenario is unreasonable and enhances epidemic growth.

\section{CONCLUSION}

The game is set, the stakes are high, and the call for action is clear. On a global scale, direct investment in diabetes prevention may reduce the economic burden of the disease. In day-to-day clinical practice, augmenting quality of life and life expectancy should be the main goals for young patients with a high metabolic risk. Therefore, when facing a patient with early clinical signs of IR, it is our responsibility to strongly and closely intervene as soon as possible.

\section{ACKNOWLEDGEMENTS}

Funding. No funding or sponsorship was received for this study or publication of this article. The article processing charges were funded by the authors. All named authors meet the International Committee of Medical Journal Editors (ICMJE) criteria for authorship, take responsibility for the integrity of the work as a whole, and have given final approval for the version to be published.

Disclosures. René Rodríguez-Gutiérrez, Alejandro Salcido-Montenegro, and José Gerardo González-González have nothing to disclose.

Compliance with Ethics Guidelines. This article is based on previously conducted studies and does not involve any new studies of human or animal subjects performed by any of the authors.

Open Access. This article is distributed under the terms of the Creative Commons Attribution-NonCommercial 4.0 International License (http://creativecommons.org/licenses/ by-nc/4.0/), which permits any noncommercial use, distribution, and reproduction in any medium, provided you give appropriate credit to the original author(s) and the source, provide a link to the Creative Commons license, and indicate if changes were made.

\section{REFERENCES}

1. GBD 2015 Disease and Injury Incidence and Prevalence Collaborators. Global, regional, and national incidence, prevalence, and years lived with disability for 310 diseases and injuries, 1990-2015: a systematic analysis for the Global Burden of Disease Study 2015. Lancet. 2016;388:1545-602.

2. GBD 2015 Mortality and Cause of Death Collaborators. Global, regional, and national life expectancy, all-cause mortality, and cause-specific mortality for 249 causes of death, 1980-2015: a systematic analysis for the Global Burden of Disease Study 2015. Lancet. 2016;388:1459-544.

3. Bommer C, Heesemann E, Sagalova V, ManneGoehler J, Atun R, Bärnighausen T, et al. The global economic burden of diabetes in adults aged 20-79 years: a cost-of-illness study. Lancet Diabetes Endocrinol. 2017;8587:1-8. 
4. Ingelfinger JR, Jarcho JA. Increase in the incidence of diabetes and its implications. $\mathrm{N}$ Engl J Med. 2017;376:1473-4.

5. Standl E, Schnell O, McGuire DK, Ceriello A, Rydén L. Integration of recent evidence into management of patients with atherosclerotic cardiovascular disease and type 2 diabetes. Lancet Diabetes Endocrinol. [Internet]. 2017;8587:1-12. http://linkinghub. elsevier.com/retrieve/pii/S2213858717300335.

6. Gómez-Flores M, González-Saldivar G, Santos-Santos O, Álvarez-Villalobos N, Rodríguez-Gutiérrez R, Tellez-Hinojosa $\mathrm{C}$, et al. Implications of a clinically ignored site of acanthosis nigricans: the knuckles. Exp Clin Endocrinol Diabetes. 2015;123:27-33.

7. Joham AE, Ranasinha S, Zoungas S, Moran L, Teede HJ. Gestational diabetes and type 2 diabetes in reproductive-aged women with polycystic ovary syndrome. J Clin Endocrinol Metab. 2014;99:447-52.

8. G S, A B, Kamath A, Shivaprakash P, Adhikari P, Up $\mathrm{R}$, et al. Acanthosis nigricansin PCOS patients and its relation with type 2 diabetes mellitus and body mass at a tertiary care hospital in southern India. J Clin Diagn Res. 2013;7:317-9.

9. González-González JG, Mancillas-Adame LG, Fernández-Reyes M, Gómez-Flores M, Lavalle-González FJ, Ocampo-Candiani J, et al. Androgenetic alopecia and insulin resistance in young men. Clin Endocrinol (Oxf). 2009;71:494-9.

10. Li WD, Fu KF, Li GM, Lian YS, Ren AM, Chen YJ, et al. Comparison of effects of obesity and non-alcoholic fatty liver disease on incidence of type 2 diabetes mellitus. World J Gastroenterol. 2015;21:9607-13. 